\title{
Allgemeines Schrifttumsverzeichnis
}

Adler/Düring/Schmaltz

Altmeppen

Bartl/Bartl/Beine/Koch/Schlarb/ Schmitt

Baumbach/Hopt

Baumbach/Hueck

Baumbach/Hueck

Bayer/Habersack (Hrsg.)

Bayer/Koch (Hrsg.)

Beck'scher Bilanz-Kommentar

Beck'sches Handbuch der GmbH

Bitter

Bitter/Heim

Bitter/Röder

Bork/Schäfer (Hrsg.)

Brandmüller

Brodmann

Brodmann

Buchegger (Hrsg.)

Centrale für GmbH (Hrsg.)

Cranshaw/Paulus/Michel (Hrsg.)

Ebenroth/Boujong/Joost/Strohn Erman

Eickhoff

Fabricius (Hrsg.)

Feine

Flume

Flume

Frankfurter Kommentar zur InsO
Rechnungslegung und Prüfung der Unternehmen, Kommentar, 6. Aufl. 1997 ff.

GmbHG, Kommentar, 10. Aufl. 2021; siehe auch Roth/Altmeppen

Heidelberger Kommentar zum GmbH-Recht, 8. Aufl. 2019

Kurzkommentar zum HGB, 39. Aufl. 2020

Kurzkommentar zum AktG, 13. Aufl. 1968

Kurzkommentar zum GmbHG, 22. Aufl. 2019

Aktienrecht im Wandel, 2007

Das neue GmbH-Recht, 2008

Handelsbilanz und Steuerbilanz. Herausgegeben von

Grottel/Schmidt/Schubert/Störk, 12. Aufl. 2020

Gesellschaftsrecht, Steuerrecht. Herausgegeben von

Prinz/Winkeljohann, 5. Aufl. 2014

Konzernrechtliche Durchgriffshaftung bei Personengesellschaften, 2000

Gesellschaftsrecht, 5. Aufl. 2020

BGB Allgemeiner Teil, 5. Aufl. 2020

Kommentar zum GmbHG, 4. Aufl. 2019

Der GmbH-Geschäftsführer im Gesellschafts-, Steuerund Sozialversicherungsrecht, 18. Aufl. 2006

Kommentar zum AktG, 1928

Kommentar zum GmbHG, 2. Aufl. 1930

Österreichisches Insolvenzrecht, Kommentar, Erster Zusatzband (BWG, GenKonkV, EKEG, VAG, URG, Insolvenz und Steuern), 2009

GmbH-Handbuch, Loseblatt

Bankenkommentar zum Insolvenzrecht, Bd. 1 und 2, 3. Aufl. 2016

Handelsgesetzbuch, Kommentar, 4. Aufl. 2020

Handkommentar zum Bürgerlichen Gesetzbuch, 16. Aufl. 2020

Die Praxis der Gesellschafterversammlung, 4. Aufl. 2006

Gemeinschaftskommentar zum Mitbestimmungsgesetz, Loseblatt

Die GmbH in Ehrenbergs Handbuch des gesamten Handelsrechts, Bd. III, 3, 1929

Allgemeiner Teil des Bürgerlichen Rechts, Bd. 1 1. Teil, Die Personengesellschaft, 1977

Allgemeiner Teil des Bürgerlichen Rechts, Bd. 12 . Teil, Die juristische Person, 1983

siehe Wimmer 


$\begin{array}{ll}\text { Gehrlein/Born/Simon (Hrsg.) } & \begin{array}{l}\text { Gesetz betreffend die Gesellschaften mit beschränkter } \\ \text { Haftung, Kommentar, 4. Aufl. 2019 }\end{array} \\ \text { Gehrlein/Witt/Volmer } & \text { GmbH-Recht in der Praxis, 4. Aufl. 2019 } \\ \text { Gersch/Herget/Marsch/Stützle } & \text { GmbH-Reform 1980, 1980 } \\ \text { Geßler/Hefermehl/Eckardt/Kropff } & \text { Aktiengesetz, Kommentar, 1974 ff. } \\ \text { Godin/Wilhelmi } & \text { Aktiengesetz, Kommentar, 4. Aufl. 1971 } \\ \text { Goette/Goette } & \text { Die GmbH, 3. Aufl. 2019 } \\ \text { Goette/Habersack (Hrsg.) } & \text { Das MoMiG in Wissenschaft und Praxis, 2009 } \\ \text { Gottwald (Hrsg.) } & \text { Insolvenzrechts-Handbuch, 5. Aufl. 2015 } \\ \text { Goutier/Seidel } & \text { Handkommentar zum GmbH-Gesetz und zur GmbH- } \\ & \text { Novelle, 1990 } \\ \text { Graf-Schlicker (Hrsg.) } & \text { InsO Kommentar, 5. Aufl. 2020 } \\ \text { Grigoleit/Rieder } & \text { GmbH-Recht nach dem MoMiG, 2009 } \\ \text { Großkommentar zum AktG } & \text { Herausgegeben von Hopt/Wiedemann, 4. Aufl. 1992 ff. } \\ & \text { Herausgegeben von Hirte/Mülbert/M. Roth, 5. Aufl. } \\ & \text { 2014 ff. } \\ \text { Großkommentar zum GmbHG } & \text { siehe Ulmer/Habersack/Löbbe und Habersack/Casper/ } \\ & \text { Löbbe } \\ \text { Großkommentar zum HGB } & \text { siehe Staub }\end{array}$

Habersack/Casper/Löbbe (Hrsg.) Gesetz betreffend die Gesellschaften mit beschränkter Haftung (GmbHG), Großkommentar. Bd. 1: 3. Aufl. 2019, Bd. 2: 3. Aufl. 2020; siehe auch Ulmer/Habersack/ Löbbe

Habersack/Henssler

Hachenburg

Hamburger Kommentar zum Insolvenzrecht

Happ

Heckschen/Heidinger (Hrsg.)

Henssler/Strohn (Hrsg.)

Hesselmann/Tillmann/MuellerThuns

Heymann

Hölters (Hrsg.)

Hoffmann/Liebs

Hüffer/Koch

Jaeger/Ziemons (Hrsg.)

Kallmeyer

Kayser/Thole (Hrsg.)

Knobbe-Keuk

Koller/Kindler/Roth/Drüen

Kölner Kommentar zum AktG

Koppensteiner

Krieger/Uwe H. Schneider (Hrsg.)

Kübler (Hrsg.)
Mitbestimmungsrecht, 4. Aufl. 2018

Großkommentar zum GmbHG. Begründet von Hachenburg, 8. Aufl. herausgegeben von Ulmer, 1990 ff. siehe Schmidt

Die GmbH im Prozess, 1997

Die GmbH in der Gestaltungs- und Beratungspraxis, 4. Aufl. 2018

Gesellschaftsrecht, Kommentar, 4. Aufl. 2019

Handbuch GmbH \& Co. KG, 22. Aufl. 2020

Handelsgesetzbuch, Kommentar, 2. Aufl. 1995 ff. Handbuch Unternehmenskauf, 9. Aufl. 2019

Der GmbH-Geschäftsführer, 3. Aufl. 2009

Aktiengesetz, Kommentar, 14. Aufl. 2020

BeckOK GmbHG

Umwandlungsgesetz, Kommentar, 7. Aufl. 2020

Heidelberger Kommentar zur InsO, 10. Aufl. 2020

Bilanz- und Unternehmenssteuerrecht, 9. Aufl. 1993

Handelsgesetzbuch, Kommentar, 9. Aufl. 2019

Herausgegeben von Zöllner, 2. Aufl. 1988 ff. Herausgegeben von Zöllner/Noack, 3. Aufl. 2004 ff. GmbH-Gesetz (Österreich), 2. Aufl. 1999

Handbuch Managerhaftung, 3. Aufl. 2017

Handbuch der Restrukturierung in der Insolvenz, 3. Aufl. 2019 
Kübler/Assmann

Kübler/Prütting/Bork (Hrsg.)

Küting/Pfitzer/Weber (Hrsg.)

Langenfeld/Miras

Liebmann/Saenger

Lutter

Lutter/Hommelhoff

Lutter/Krieger/Verse

Lutter/Scheffler/U. H. Schneider (Hrsg.)

Lutter/Ulmer/Zöllner (Hrsg.)

Meyer-Landrut/Miller/Niehus

Michalski/Heidinger/Leible/

J. Schmidt

Münchener Anwaltshandbuch GmbH-Recht

Münchener Handbuch des Gesellschaftsrechts

Münchener Kommentar zum
AktG
Münchener Kommentar zum
BGB

Münchener Kommentar zum GmbHG

Münchener Kommentar zum HGB

Münchener Kommentar zur InsO

Münchener Kommentar zur ZPO

Nerlich/Römermann (Hrsg.)

Obermüller

Palandt

Raiser/Veil

Raiser/Veil/Jacobs

Reich-Rohrwig

Röhricht/Graf von Westphalen/ Haas (Hrsg.)
Gesellschaftsrecht, 6. Aufl. 2006

InsO, Kommentar zur Insolvenzordnung, Loseblatt

Handbuch der Rechnungslegung, Loseblatt

GmbH-Vertragspraxis, 8. Aufl. 2019

Kommentar zum GmbHG, 7. Aufl. 1927

Umwandlungsgesetz. Herausgegeben von Bayer/J. Vetter, 6. Aufl. 2019

GmbH-Gesetz, Kommentar, 20. Aufl. 2020

Rechte und Pflichten des Aufsichtsrats, 7. Aufl. 2020

Handbuch der Konzernfinanzierung, 1998

Festschrift 100 Jahre GmbHG, 1992

Kommentar zum GmbH-Gesetz, 1987

GmbHG, 3. Aufl. 2017

Herausgegeben von Römermann, 4. Aufl. 2018

Band 2: Kommanditgesellschaft, GmbH \& Co. KG, Publikums-KG, Stille Gesellschaft. Herausgegeben von Gummert/Weipert, 5. Aufl. 2019; Band 3: Gesellschaft mit beschränkter Haftung. Herausgegeben von Priester/ Mayer/Wicke, 5. Aufl. 2018; Band 4: Aktiengesellschaft. Herausgegeben von Hoffmann-Becking, 5. Aufl. 2020 Herausgegeben von Goette/Habersack, 4. Aufl. 2014 ff., 5. Aufl. $2019 \mathrm{ff}$.

Herausgegeben von Rixecker/Säcker/Oetker, 6. Aufl. 2012 ff. Herausgegeben von Säcker/Rixecker/Oetker/ Imperg, 7. Aufl. 2015 ff., 8. Aufl. 2019 ff.

Herausgegeben von Fleischer/Goette, Bd. 1: 3. Aufl. 2018, Bd. 2: 3. Aufl. 2019, Bd. 3: 3. Aufl. 2018

Herausgegeben von Karsten Schmidt, 4. Aufl. 2016 ff.

Herausgegeben von Stürner/Eidenmüller/Schoppmeyer, 4. Aufl. $2019 \mathrm{ff}$.

Herausgegeben von Krüger/Rauscher, 5. Aufl. 2016 f.

Insolvenzordnung, Loseblatt

Insolvenzrecht in der Bankpraxis, 9. Aufl. 2016

Bürgerliches Gesetzbuch, Kommentar, 79. Aufl. 2020

Recht der Kapitalgesellschaften, 6. Aufl. 2015

Kommentar zum Mitbestimmungsgesetz und Drittelbeteiligungsgesetz, 7. Aufl. 2019

Das österreichische $\mathrm{GmbH}$-Recht in systematischer

Darstellung, 1983; 2. Aufl., Bd. I, 1997

HGB, Kommentar, 5. Aufl. 2019 
Römermann/Wachter (Hrsg.)

Roth/Altmeppen

Rowedder/Schmidt-Leithoff

Saenger/Inhester (Hrsg.)

Schimansky/Bunte/Lwowski (Hrsg.)

Schlegelberger

Schlegelberger/Quassowski

A. Schmidt (Hrsg.)

Karsten Schmidt

Karsten Schmidt

Karsten Schmidt (Hrsg.)

Karsten Schmidt/Lutter (Hrsg.)

Karsten Schmidt/Uhlenbruck (Hrsg.)

Soergel

Staub

Staudinger

Tillmann/Mohr

Tillmann/Schiffers/Wälzholz/ Rupp

Uhlenbruck

Ulmer/Habersack/Löbbe

Vogel

Wicke

Widmann/Mayer

Wiedemann

Wimmer (Hrsg.)

Wißmann/Kleinsorge/Schubert

Wünsch

Würdinger

Zöller
GmbH-Beratung nach dem MoMiG, GmbHR-Sonderheft 2008 (Oktober)

GmbHG, Kommentar, 9. Aufl. 2019; siehe auch

Altmeppen

Kommentar zum GmbH-Gesetz, 6. Aufl. 2017

GmbHG, Kommentar, 4. Aufl. 2020

Bankrechts-Handbuch, 5. Aufl. 2017

Kommentar zum HGB, 5. Aufl. 1973 ff.

Kommentar zum Aktiengesetz 1937, 3. Aufl. 1939

Hamburger Kommentar zum Insolvenzrecht, 7. Aufl.

2019

Gesellschaftsrecht, 4. Aufl. 2002

Handelsrecht, 6. Aufl. 2014

Insolvenzordnung, Kommentar, 19. Aufl. 2016

AktG, 4. Aufl. 2020

Die GmbH in Krise, Sanierung und Insolvenz,

5. Aufl. 2016

BGB, Kommentar, 13. Aufl. 1999 ff.

Großkommentar zum Handelsgesetzbuch. Herausgegeben von Canaris/Habersack/Schäfer, 5. Aufl. 2009 ff.

Kommentar zum Bürgerlichen Gesetzbuch, 2003 ff.

GmbH-Geschäftsführer, 11. Aufl. 2020

Die GmbH im Gesellschafts- und Steuerrecht,

6. Aufl. 2015

Insolvenzordnung, Kommentar, 15. Aufl. 2019 Gesetz betreffend die Gesellschaften mit beschränkter Haftung (GmbHG), Großkommentar. Bd. 3: 2. Aufl. 2016, siehe auch Habersack/Casper/Löbbe

Kommentar zum GmbHG, 2. Aufl. 1956

Gesetz betreffend die Gesellschaften mit beschränkter

Haftung (GmbHG), 4. Aufl. 2020

Umwandlungsrecht, Kommentar, Loseblatt

Gesellschaftsrecht, Bd. 1: Allgemeine Grundlagen, 1980,

Bd. 2: Recht der Personengesellschaften, 2004

Frankfurter Kommentar zur InsO, 9. Aufl. 2018

Mitbestimmungsrecht, 5. Aufl. 2016

Kommentar zum GmbHG (Österreich), 1988

Aktienrecht und das Recht der verbundenen Unter-

nehmen, 4. Aufl. 1981

Zivilprozessordnung, 33. Aufl. 2020 